\title{
Energy Efficiency in Passive Optical Networks: Where, When, and How?
}

\author{
Luca Valcarenghi, Dung Pham Van, Pier Giorgio Raponi, and Piero Castoldi, \\ Scuola Superiore Sant'Anna \\ Divanilson R. Campelo, Federal University of Pernambuco (UFPE) \\ Shing-Wa Wong, She-Hwa Yen, and Leonid G. Kazovsky, Stanford University \\ Shinii Yamashita, Fujitsu Laboratories Ltd.
}

\begin{abstract}
This article provides an overview of current efforts in reducing energy consumption in passive optical access networks. Both ITU-T and IEEE standardized PONs are considered. The current solutions proposed by standardization authorities, industry, and academia are classified based on the layer they address in the standardized architectures: physical layer, data link layer, and hybrid. Then, the article provides answers to major questions, such as where, when, and how to reduce PON energy consumption in TDM PONs by means of a quantitative evaluation. Results show that to reduce energy consumption, ONUs must be provided with physical devices that are not only power-efficient but also provide improved services (e.g., fast synchronization) to upper layers. For this latter purpose, novel physical ONU architectures are proposed to speed up the synchronization process and enable effective data link layer solutions. Finally, the feasibility of switching ONUs to low power mode in idle slots is assessed through a testbed implementation.
\end{abstract}

ommunications network energy efficiency is currently drawing a lot of attention. Not only is greenhouse gas emission reduction sought worldwide, but also carriers long to decrease their operating expenditures. Previous research reported that access networks (fixed and mobile) are the major contributors to current communications network energy consumption [1]. This is mainly due to the large number of involved elements (i.e., the customer premises equipment - CPE). The replacement of digital subscriber line (DSL)-based wired access networks with optical access systems, such as passive optical networks (PONs), has the potential of reducing the energy per bit consumed by access networks. However, optical access networks are forecast to be the largest contributors to wired optical communication network energy consumption for the next 10 years yet [2].

Within PONs, CPE (i.e., optical network units - ONUs) are the energy hungriest devices. Indeed, they are numerous and often idle because of the current low utilization of PON capacity and the shared medium architecture, but "always on." In [2] and references therein, it is shown that ONUs contribute over 65 percent of the total PON power consumption. In other words, ONUs are the major target for energy saving in PONs, and their energy efficiency will be much improved if idle ONUs can be properly turned off.

This work was supported in part by the the U.S.-Italy Fulbright Research Scholar Program, U. S. National Science Foundation (NSF) under Grant no. 0627085, Brazilian National Council for Scientific and Technology Development $(\mathrm{CNPq})$, and OPNET university program.
This article first surveys ONU energy consumption in PONs standardized by both the International Telecommunication Union - Telecommunication Standards Sector (ITU-T) and IEEE. Then it describes solutions currently proposed to reduce ONU energy consumption by standardization authorities, industry, and academia. The current solutions are classified into three categories: physical layer, data link layer, and hybrid. Then, by considering a time-division multiplexed (TDM) PON, the article provides hints on the suitability of specific solutions through a quantitative evaluation. Throughout the classification method and quantitative evaluation, it is possible to understand where, when, and how to intervene in ONU architecture or its operations to reduce energy consumption.

In particular, a data link layer solution based on turning off (i.e., switching to low power or sleep mode) the ONU outside of its allocated slots is considered. Novel physical ONU architectures are proposed to provide the upper layer with faster synchronization (i.e., physical layer services). This allows ONUs to avoid very early wake up after a sleep period, thus increasing energy efficiency. Finally, the feasibility of the proposed data link layer solution is assessed by means of its implementation in a testbed.

\section{Power Consumption in Current Passive Optical Networks}

Currently standardized PONs are time-division multiple access (TDMA) PONs, in which the only active devices reside in the central office (i.e., the optical line terminal, OLT) and 


\begin{tabular}{|c|c|c|c|c|c|c|c|c|}
\hline \multirow{2}{*}{$\begin{array}{l}\text { Receiver front-end } \\
\text { component }\end{array}$} & \multicolumn{2}{|c|}{ EPON } & \multicolumn{2}{|c|}{ GPON } & \multicolumn{2}{|c|}{ 10G-EPON } & \multicolumn{2}{|c|}{ XG-PON } \\
\hline & Avg & Range & Avg & Range & Avg & Range & Avg & Range \\
\hline TIA & 83.4 & $56-112$ & 83.4 & $56-112$ & 123 & $105-160$ & 123 & $105-160$ \\
\hline CDR & 545 & $540-580$ & 520 & $260-790$ & 356 & NA & 356 & NA \\
\hline SERDES & 550 & $530-660$ & 560 & $530-660$ & \multirow{2}{*}{\multicolumn{2}{|c|}{ NA }} & \multirow{2}{*}{\multicolumn{2}{|c|}{ NA }} \\
\hline $\begin{array}{l}\text { Total receiver front- } \\
\text { end }\end{array}$ & \multicolumn{2}{|c|}{1302} & \multicolumn{2}{|c|}{1292} & & & & \\
\hline Back-end circuit & \multicolumn{2}{|c|}{2700} & \multicolumn{2}{|c|}{3150} & \multicolumn{2}{|r|}{5850} & \multicolumn{2}{|c|}{6750} \\
\hline Whole ONU (services) & \multicolumn{2}{|c|}{$\begin{array}{c}6000 \\
\text { (Ethernet data } \\
\text { port+IPTV) }\end{array}$} & \multicolumn{2}{|c|}{$\begin{array}{c}7000 \\
\text { (triple play }+ \text { multicast } \\
\text { video) }\end{array}$} & \multicolumn{2}{|c|}{$\begin{array}{c}13,000 \\
\text { (prediction) }\end{array}$} & \multicolumn{2}{|c|}{$\begin{array}{c}15,000 \\
\text { (PoE on Gigabit } \\
\text { Ethernet port) }\end{array}$} \\
\hline
\end{tabular}

Table 1. Power consumption [mW] of discrete components in ONU.

at the customer premises (i.e., the ONU). PONs are typically deployed in a tree topology, in which a passive optical splitter is used as the remote node (RN) to broadcast signals from the OLT to the ONUs. Signals from different ONUs are multiplexed in time to share the upstream wavelength. Today's widespread PON standards are the Gigabit-capable PON (GPON ITU-T Rec. G.984.x) with capacity of $1 \mathrm{~Gb} / \mathrm{s}$, the 10Gb-capable PON (XG-PON ITU-T Rec. G.987.x) with capacity of $10 \mathrm{~Gb} / \mathrm{s}$, the Ethernet PON (EPON IEEE 802.3-2008 section 5) with capacity of $1 \mathrm{~Gb} / \mathrm{s}$, and $10 \mathrm{~Gb} / \mathrm{s}$ Ethernet PON (10G-EPON, IEEE 802.3av-2009) with capacity of $10 \mathrm{~Gb} / \mathrm{s}$. Typically, an ONU consists of a transceiver and of electronic circuitry that implements the medium access control (MAC) layer functions, the so called system on chip (SoC). The transceiver building blocks consist of both optical and electronic components. The optical components are:

- A transmitter, typically a Fabry-Perot (F-P) laser, which optically transmits data to the OLT in the upstream direction

- An avalanche photo-diode (APD), which receives data from the OLT

- A WDM coupler, which couples downstream and upstream wavelengths into a single optical fiber.

The electronic section of the transceiver includes:

- A burst-mode laser driver (BM-LD) to drive the F-P laser for upstream transmission

- A trans-impedance amplifier (TIA) to translate and amplify downstream photocurrent into voltage

- A limiting post-amplifier to reshape the voltage outcoming from the TIA ${ }^{1}$

- A clock and data recovery (CDR) system

Several implementations of the ONU transceiver are available with different degrees of integration and thus different power consumption levels. The main functional block of the electronic circuitry is the serializer-deserializer (SERDES), which converts serial signals to parallel signals and vice versa. In this article, the remaining functions of the $\mathrm{SoC}$ are included in a generic back-end electronic circuit.

${ }^{1}$ The TIA and post-amplifier perform $2 R$ (re-amplification and reshaping) signal regeneration.
ONU power consumption data are available from component data sheets, research papers, and standards [3]. Table 1 summarizes power consumption data for EPON, GPON, 10GEPON, and XG-PON. Data have been collected by analyzing the data sheets available online from tens of vendors. Table 1 shows that the CDR and the SERDES are the ONU components that consume the highest power in both EPON and GPON - they consume more than 80 percent of the power of the entire ONU receiver front-end. Although data are not available for $10 \mathrm{G}-\mathrm{EPON}$ and XG-PON, a similar behavior is expected. However, the SERDES is commonly shared by the ONU receiver and transmitter. Moreover, the collected data confirm what presented in [4]: between $60-70$ percent of the overall ONU power consumption is due to the PON transceiver and back-end electronic circuit.

\section{Solutions for Saving Energy in Passive Optical Networks}

This section provides a classification of the solutions proposed so far by standardization authorities (i.e., ITU-T and IEEE), industry, and academia for implementing energy-efficient optical access networks, and highlights their characteristics. The solutions are classified based on the architectural layer in which they are implemented: physical layer, data link layer, and hybrid. This classification is consistent with both the common GPON layers defined in ITU-T Recommendation G.984.1 and the IEEE 802.3-2008 layered architecture.

\section{Physical Layer Solutions}

Physical layer solutions aim at reducing PON energy consumption by targeting the physical layer of PON architectures without necessarily modifying the upper layer protocols. They can be further divided into device-oriented and service-oriented. Device-oriented solutions aim at reducing the energy consumption of the physical devices. Service-oriented solutions aim at improving the performance of the services provided by the physical layer to enable upper layer solutions (e.g., sleep mode). They are often utilized in combination with data link layer solutions to implement hybrid solutions. 


\begin{tabular}{|c|c|c|c|c|}
\hline \multirow{2}{*}{ Function } & \multicolumn{2}{|c|}{$\begin{array}{c}\text { Tier 2011-2012 } \\
1.1 .2011-31.12 .2012\end{array}$} & \multicolumn{2}{|c|}{$\begin{array}{c}\text { Tier 2013-2014 } \\
1.1 .2013-31.12 .2014\end{array}$} \\
\hline & Idle-state & On-state & Idle-state & On-state \\
\hline Central functions plus WAN interface & 5.6 & 8.8 & 5.3 & 7.7 \\
\hline $1 \mathrm{GE}$ interface & 0.3 & 0.9 & 0.2 & 0.6 \\
\hline 2 FXS (only one interface is "on" in idle-state) & $1 \times 0.5$ & $2 \times 1.5=3.0$ & $1 \times 0.3$ & $2 \times 1.2=2.4$ \\
\hline $1 \mathrm{MoCA}$ & 2.0 & 2.5 & 1.8 & 2.2 \\
\hline Total & 8.4 & 15.2 & 7.6 & 12.9 \\
\hline
\end{tabular}

Table 2. Target power consumption [W] of 10G-EPON ONU (1GE, 2 FXS, 1 MoCA).

The implementation of device-oriented physical layer solutions is strongly advised by standardization authorities and European Union (EU) sponsored research centers. For example, the Institute for Energy of the Joint Research Center of the European Commission Directorate General has published a "Code of Conduct on Energy Consumption of Broadband Equipment" (BBCoC) [5]. In the BBCoC, forthcoming year power consumption targets for both $\mathrm{CPE}$ and network equipment are provided. In the $\mathrm{BBCoC}$, three CPE operation states are identified: on-state, off-state, and idle-state. A device is in the on-state if all of its components (i.e., interfaces) are fully operational, transmitting and receiving a minimum amount of traffic. A device is in the off-state if all of its interfaces are not providing any function. A device is in the idle-state if all of its interfaces are not processing or transmitting a significant amount of traffic. The BBCoC considers EPON, GPON, 10GEPON, and XG-PON. By utilizing the data provided in [5] it is possible to compute the target power consumption of a typical 10G-EPON ONU with one Gigabit Ethernet interface, two Foreign Exchange Station (FXS) interfaces, ${ }^{2}$ and one Multimedia over Coax Alliance (MoCA) interface. The values, summarized in Table 2, show that even if the targets are met, more than 50 percent of the power budget is consumed by the ONU central functions (which also include Gigabit Ethernet switch functionalities) and the wide area network (WAN) interface.

Within the IEEE P802.3az EEE task force (now closed), a method called adaptive link rate (ALR) was under discussion [6]. ALR is based on adapting the interface line rate to the network traffic to save power. ALR is based on the fact that transmitting at low rates requires less power than transmitting at high rates. This is similar to dynamic frequency scaling (DFS), commonly utilized in microprocessors in combination with dynamic voltage scaling (DVS), which is capable of achieving a cubic reduction in dynamic power consumption [7]. However, ALR was found to be quite complex to implement, and it has not been standardized yet.

In the electronic industry as well as in the optical one, component integration is the main device-oriented physical layer solution to reduce energy consumption. As shown in Table 3 (top), electronic integration decreases component power consumption. For example, the integration of CDR, LA, and SERDES allows power consumption to be almost halved with respect to utilizing discrete components in EPON.

Several patent applications proposing device-oriented physical layer solutions have also been submitted. For instance, in [8] a low-power sleep logic circuit is proposed that extends the

${ }^{2}$ In this configuration it is assumed that voice over IP is utilized for telephone services. battery lifetime of a GPON optical network terminal (ONT) after the AC main power has failed. The low-power logic circuit monitors the main external power source and the battery status after mains failure, and it selectively switches off ONU interfaces based on the external power source and battery status.

Novel devices have been proposed in academia for extending the reach of next-generation PONs. Such devices can also be exploited as device-oriented physical layer solutions for decreasing the PON power budget. For example, the utilization of a quasi-passive optical switch [9] at the PON remote node (RN) allows the RN splitting loss to be reduced and thus the power transmitted by the OLT. However, this solution decreases the required optical power budget only; hence, the benefits in terms of energy consumption are not expected to be high.

Electronic integration can also be exploited as a service-oriented physical layer approach. Indeed, Table 3 (bottom) shows that the integration of LA, CDR, and SERDES can shorten clock recovery time $T_{\text {rec }}$ to almost one fourth that of a discrete CDR.

A service-oriented physical layer solution that utilizes different modulation formats for allowing faster clock recovery while the ONU exits from sleep mode has been proposed in academia [10]. The utilization of Manchester modulation format instead of the standard non-return to zero (NRZ) format is capable of allowing faster clock recovery in commercial CDR boards. However, Manchester modulation pulse shape is not within the standard mask of the transmitter eye diagram specified in IEEE 802.3av-2009. Optically powered fiber networks [11] can also be exploited to implement service-oriented physical layer solutions. In optically powered fiber networks, remote devices (e.g., sensors) accumulate and store energy within their idle time, and then perform their functions for a short time and send the acquired information. Similar solutions can be applied for remotely powering up a control circuit that wakes up ONUs after a sleep period.

\section{Data Link Layer Solutions}

Data link layer solutions target the data link layer of the IEEE 802.3 architecture (i.e., the medium access control [MAC] layer) or the transmission convergence (TC) layer in GPON (ITU-T Recommendation G.984.3) and XG-PON (ITU-T Recommendation G.987.3). They are based on the possibility of switching network elements to a low power mode (e.g., sleep mode). In GPON, similar functionalities are also defined in the ONU management and control interface layer (OMCI) (ITU-T Recommendation 984.4 for GPON and ITU-T Recommendation G.988 for XG-PON).

Even though low power mode support is required in the ONUs physical layer, data link layer solutions are based only 


\begin{tabular}{ccccc}
\hline \multirow{2}{*}{ Component } & EPON & GPON & 10G-EPON & XG-PON \\
\cline { 2 - 5 } & \multicolumn{4}{c}{ Power [mW] } \\
\hline CDR & 545 & 520 & 356 & 356 \\
\hline CDR+LA & 410 & 410 & 350 & NA \\
\hline CDR+SERDES & 910 & 790 & NA & \\
\hline CDR+LA+SERDES & 610 & 610 & & \\
\hline
\end{tabular}

\begin{tabular}{|c|c|c|c|c|c|c|c|c|}
\hline \multirow{3}{*}{ Component } & \multicolumn{8}{|c|}{$T_{r e c}[\mathrm{~ms}]$} \\
\hline & \multicolumn{2}{|c|}{ EPON } & \multicolumn{2}{|c|}{ GPON } & \multicolumn{2}{|c|}{ 10G-EPON } & \multicolumn{2}{|c|}{ XG-PON } \\
\hline & Avg & Range & Avg & Range & Avg & Range & Avg & Range \\
\hline \multicolumn{9}{|c|}{ Discrete components } \\
\hline CDR & 2.05 & $0.6-14$ & 2.2 & $0.6-14$ & 1.1 & $0.2-2$ & 1.1 & $0.2-2$ \\
\hline \multicolumn{9}{|c|}{ Integrated components } \\
\hline $\mathrm{CDR}+\mathrm{LA}$ & 1 & & 1 & & 2 & & NA & \\
\hline CDR + DMUX & 3 & & 2 & & NA & & NA & \\
\hline $\mathrm{LA}+\mathrm{CDR}+\mathrm{DMUX}$ & 0.6 & & 0.6 & & NA & & NA & \\
\hline
\end{tabular}

Table 3. Power consumption reduction (top) and CDR time ( $\mathrm{T}_{\mathrm{rec}}$ ) reduction (bottom) through integration.

on extensions of the Multipoint Control Protocol (MPCP), the Physical Layer Operation, Administration, and Management (PLOAM) protocol, or the ONT management communication channel (OMCC), and on modified dynamic bandwidth allocation (DBA) algorithms.

ITU-T G.Sup45 [3] proposes three types of power conservation methods: power shedding, dozing, and sleeping (further divided into deep and fast sleep). The methods mainly differ in the behavior of the ONU transmitter and receiver. Power shedding powers off or reduces power to non-essential functions and services (e.g., interfaces) while maintaining a fully operational optical link. Dozing powers off the ONU transmitter when the ONU does not have upstream traffic to transmit while keeping the ONU receiver "always on." Deep sleep turns off the whole ONU transceiver and all the ONU functions and services, except activity detection. In deep sleep, the ONU loses the incoming downstream traffic and may wake up only when the ONU is switched "on" on local stimulus or at the expiration of a locally maintained timer. Fast sleep is based on sleep cycles that alternate sleep periods, when the whole ONU transceiver is turned off together with all the non-essential functions, and active periods, when the optical transceiver and the necessary supporting functions are turned on. The OLT learns the capabilities of the ONU to support a specific power saving mode through extensions of the management channel, and it can also negotiate with the ONU which modes to select. Transitions to and from a particular power save mode of the ONU can be supported by different signaling messages, such as a PLOAM Power Save Mode (PSM) message or by the OMCI framework as proposed in ITU-T Recommendation G.988. The qualitative comparison in [3] shows that the OMCI signaling is the most functionally powerful method, and it does not require TC layer modification.
On the other hand, PLOAM PSM signaling has the deepest impact on the TC layer (introduction of a new PLOAM message), but it may achieve faster completion of the mode transition. In [4], it is shown that the combination of sleeping and power shedding has the potential to reach 80 percent power savings. However, in the case of power shedding, all the packets arriving to the ONU user-network interface (UNI) are lost. Thus, power shedding can be applied only during long times of ONU inactivity. Moreover, even G.Sup45 proposes a near-term solution based on power shedding. The solution allows operators to turn off ONU services during the times of day when they are not needed.

Although IEEE 802.3az includes the support of Low Power Idle (LPI) mode for point-to-point links, EPON and $10 \mathrm{G}-\mathrm{EPON}$ do not contain any specification of data link layer solutions for energy consumption reduction in PONs. Methods for enhancing MPCP with sleep mode control messages have been proposed, but not standardized yet [12].

Several vendors are also seeking data link layer solutions for reducing ONU energy consumption. This is witnessed by the large number of patent applications that have been recently submitted. For example, in [12] a cyclic sleep mode is proposed to be implemented in IEEE 802.3 style networks. Both OLT driven and ONU driven methods are considered, but the final decision of switching to sleep mode is taken by the OLT. Extension to the EPON MPCP protocols to implement the handshake between the OLT and the ONU to switch to sleep mode are also proposed that introduce new messages such as SLEEP, SLEEP_REQ, CHANGE_SLEEP_MODE. Many vendors are also advertising the introduction of power saving modes into their devices. In these cases, the ONU supports power saving modes for power consumption reduction during power outages and standby periods.

In [13] two data link layer solutions are presented for reducing energy consumption of EPON and $10 \mathrm{G}-\mathrm{EPON}$. The less aggressive solution defines sleep-mode control and sleepaware traffic scheduling to allow ONUs to sleep over more than one DBA cycle. The more aggressive solution allows the ONU to sleep within one DBA cycle. In both solutions, four ONU power levels are defined based on the possibility of selectively turning off the ONU transmitter or receiver. If both the transmitter and receiver are off, even the ONU MAC and SERDES are turned off. In sleep for more than one DBA cycle solution, the transmitter or receiver are turned off if no upstream/downstream traffic exists. In sleep within one DBA cycle solution, the ONU transmitter goes to sleep outside its assigned upstream slot. The ONU receiver sleep time, instead, is based on downstream traffic scheduling performed by the OLT. The main issues are making the ONU aware of the scheduling to avoid early or late wake up ONU. Early wake up implies reduced energy savings, while late wake up implies additional delay. 


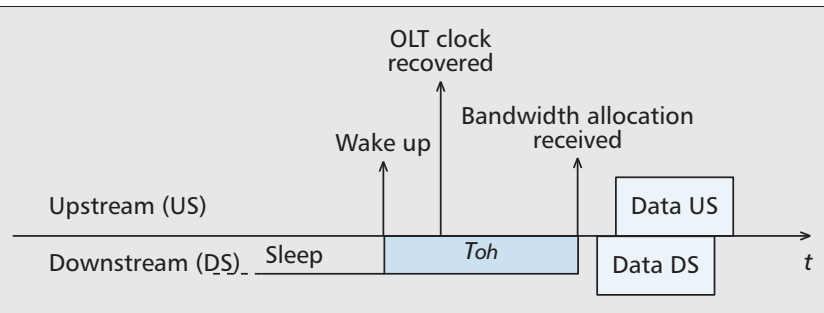

(a)

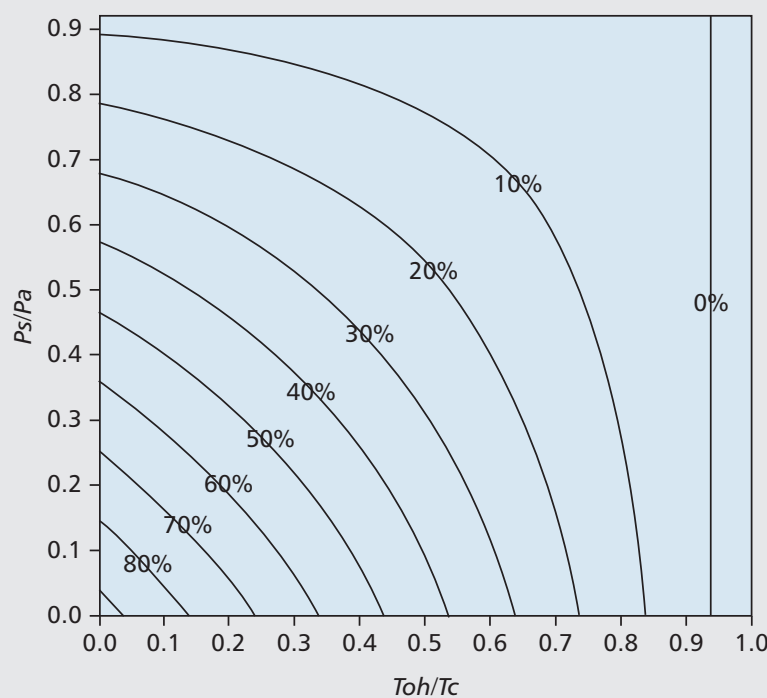

(b)

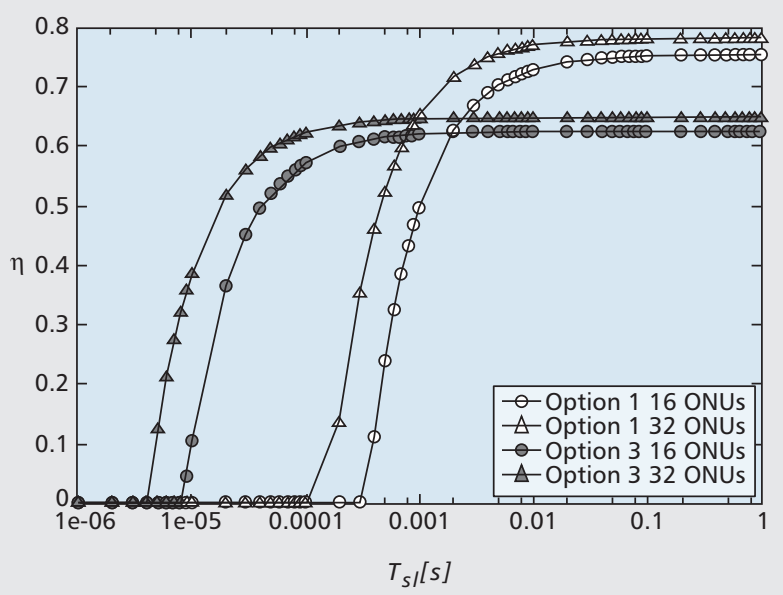

(c)

Figure 1. TDM EPON with sleep mode overhead and energy saving trade-off: a) ONU synchronization overhead; b) energy saving percentage $\eta$ as a function of $\mathrm{T}_{\mathrm{oh}} / \mathrm{T}_{\mathrm{c}}$ and $\mathrm{P}_{\mathrm{S}} / \mathrm{P}_{\mathrm{a}}$ in TDM PON with sleep mode; c) energy savings percentage in option 1 and option 3 from [16] in TDM PON with sleep mode.

In [14] the performance evaluation of two power saving methods standardized in XG-PON, cyclic sleep (fast sleep) and doze mode, is performed through simulations. One of the key features in XG-PON power saving modes is the utilization of timers to regulate the duration of sleep periods. This solution simplifies the coordination between ONU and OLT because timers can be updated but not necessarily for each sleep period. Another feature is the utilization of the sleep (or doze) aware state in which an ONU periodically (i.e., based on timers) wakes up to collect potential wake-up messages from the OLT. In addition to the timers, the XG-PON power saving solution utilizes scheduling messages to schedule ONU into sleep or doze mode. Simulations performed in [14] show that in cyclic sleep, a longer sleep period (determined by the value of the timer $T_{\text {sleep }}$ ) allows for larger energy consumption reduction. However, if the traffic is low and the $T_{\text {sleep }}$ is large, the delay of downstream message is very long (close to $90 \mathrm{~ms}$ ). On the contrary, upstream traffic delay is not affected by the sleep duration because of the ONU initiated wake-up that does not depend on $T_{\text {sleep }}$. In doze mode, the absence of delay impairments on the downstream traffic is obtained at the expense of reduced power saving. However, no upper bound is necessary for $T_{\text {sleep }}$, resulting in the possibility of longer sleep periods with, in turn, similar or even higher energy savings than cyclic sleep.

\section{Hybrid Solutions}

Hybrid solutions are those that combine physical and data link layer solutions to reduce energy consumption. One hybrid solution is proposed in [15] for 10G-EPON where EPON and 10G-EPON OLTs and ONUs coexist. The approach is based on combining sleep and periodic wake-up (SPW) (i.e., cyclic sleep in G.Sup45 terminology) and adaptive link rate (ALR).
In SPW, the OLT requests an ONU to switch to sleep mode because of the absence of downstream traffic. The OLT sets the ONU sleeping time, after which the ONU wakes up to request the OLT whether it can sleep further or not. In the ALR the downlink rate is switched between 1 and $10 \mathrm{~Gb} / \mathrm{s}$ based on the amount of traffic. It is again the OLT issuing a control message to the ONU for switching between 1 and 10 $\mathrm{Gb} / \mathrm{s}$ downstream transmission. The ONU replies with an acknowledgment message after having performed the receiver switching. Simulation results show that the hybrid mechanism is capable of providing larger power saving than the two methods in isolation. In addition, in [15] the successful implementation of the proposed method in a testbed is shown.

Another possible hybrid solution could be provided by combining MPCP protocol extensions for sleep mode, such as cyclic sleep [12], with physical layer approaches to remotely power the ONU [11]. In this way, the ONU wake up is directly triggered by the OLT by powering the ONU up.

Other studies performed by the authors of this article propose the combination of modified ONU architectures with sleep mode during ONU idle slots for improving the clock recovery after the ONU wake up. More details on these solutions are summarized in the following section.

\section{Where, When, and How to Save Energy in PONs}

The energy efficiency of a PON can be largely improved by turning off the non-essential functions or components inside an ONU whenever it is idle. For instance, ONUs are "on" but idle when the downstream traffic is not destined to them and 


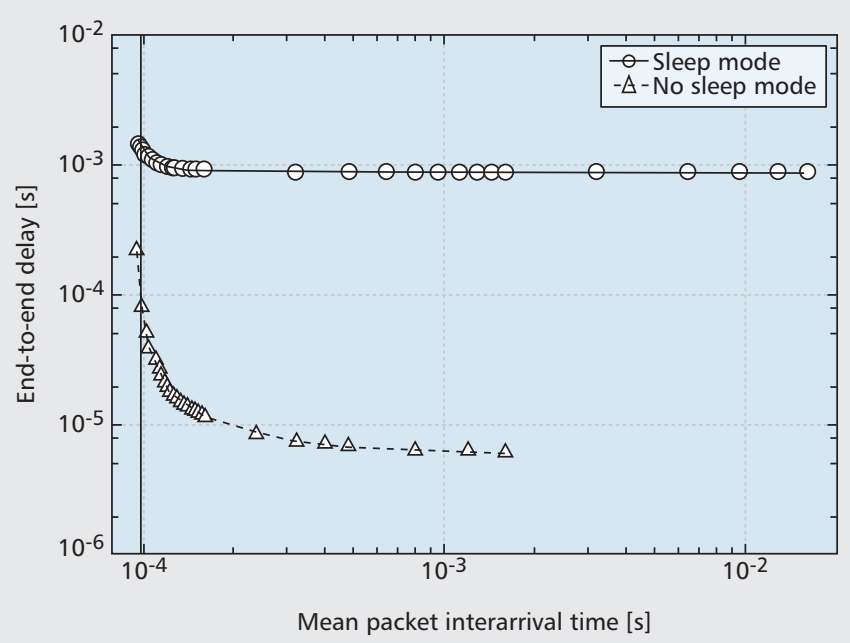

(a)

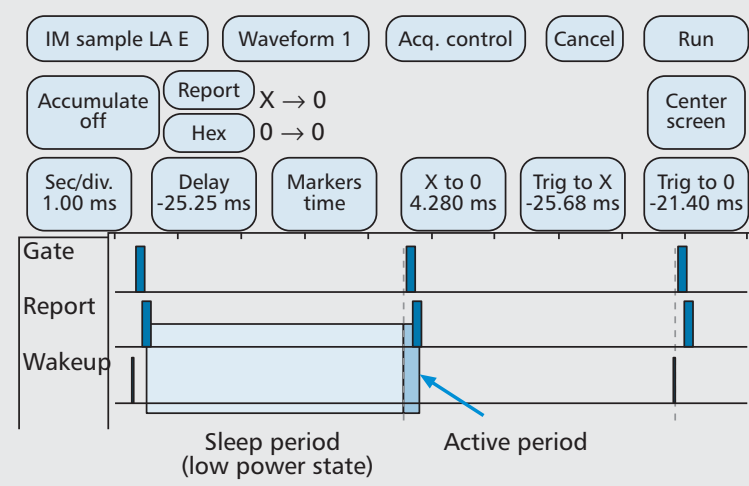

(b)

Figure 2. TDM EPON with sleep mode performance evaluation through simulation and experimental testbed: a) average end-to-end delay as a function of mean packet interarrival time in TDM EPON with sleep mode; and b) testbed results.

they are not transmitting data upstream. Basically, this helps answering the questions where and when energy can be saved in PON, at a general level. The hints for more relevant answers to these basic but critical questions can be found in this section where a quantitative evaluation for EPON in which TDM is used for both upstream and downstream transmission (i.e., PON with fixed bandwidth allocation) is performed.

In this scenario, referred to as TDM PON with sleep mode, ONUs can be turned off (i.e., switched to sleep mode) outside their assigned transmission slot and can be "off" for a period of time longer than one DBA cycle. Assume that $P_{a}$ is the power consumed by the ONU when active (i.e., when "on"), while $P_{S}$ is the power consumed in low power mode (i.e., while the ONU is sleeping or "off"). The energy utilized by one ONU to receive the same $D$ bits of data without sleep mode $E$ and with sleep mode $E^{\prime}$ can be written as

$$
\begin{aligned}
& E=T_{c} P_{a} \\
& E^{\prime}=\left(T_{s l}+T_{o h}\right) P_{a}+\left(T_{c}-T_{s l}-T_{o h}\right) P_{s},
\end{aligned}
$$

where $T_{s l}$ is the slot time (i.e., the time allocated to each ONU for transmission/reception), $T_{c}$ is the DBA cycle time (i.e., the time between two consecutive transmission/reception by the same ONU), and $T_{o h}$ is the time taken by the ONU to recover clock and data synchronization upon waking up or overhead time ${ }^{3}$ (as depicted in Fig. 1a).

By considering TDM PON with $N$ ONUs in the system and assuming the guard time between two consecutive time slots is negligible, it follows that $T_{s l}=T_{c} / N$ and the energy saving percentage $\eta$ can be expressed as

$$
\eta=\frac{E-E^{\prime}}{E}=\frac{N-1}{N}+\frac{T_{o h}}{T_{c}} \frac{P_{S}}{P_{a}}-\frac{T_{o h}}{T_{c}}-\frac{N-1}{N} \frac{P_{S}}{P_{a}} .
$$

In Eq. 3, $T_{o h} / T_{c}$ is defined as the ratio between the overhead time and the cycle time, or time ratio, and $P_{s} / P_{a}$ is defined as the ratio between the power consumption of an

\footnotetext{
${ }^{3}$ In both EPON and GPON, if a receiver is turned off its clock drifts. Upon waking up, the ONU must recover synchronization with both the OLT clock and the network scheduling.
}

ONU in sleep mode and that in an active state, or power ratio. The time and power ratios are two key parameters that affect the power savings in TDM PONs with sleep mode.

Equation 3 helps understanding when and how energy is saved. Figure 1b depicts results obtained for a PON with $N=$ 16 and $T_{c}=2 \mathrm{~ms}$, and it shows that a given energy saving percentage can be achieved by reducing the time ratio or the power ratio or both. However, given a specific ratio (e.g., power ratio), all the savings are not achievable even if the other ratio (e.g., the time ratio) is decreased to zero. For example, even if the ONU power consumption is reduced by half, achievable energy savings do not reach 50 percent. Major savings can be obtained only if both ratios are reduced. For example, to obtain at least 50 percent savings, the power ratio must be less than 0.47 and the time ratio must be less than 0.43 . Therefore, even though components with very low power consumption are developed, if the ONU cannot recover synchronization quickly, high energy savings cannot be achieved. The opposite is also true. Furthermore, with a fixed target ratio, it is possible to choose which components (i.e., where) inside the ONU must be turned off to achieve it.

To have a more detailed answer to the question of how energy can be saved, Eq. 3 can be linked to the energy-efficient solutions discussed in the previous section. More concretely, the energy consumption of an ONU can be reduced by either minimizing the recovery time of the CDR circuit for decreasing the time ratio or designing an efficient sleep scheduler that puts more components of an ONU to sleep for decreasing the power ratio. The former approach can be classified as a service-oriented physical layer solution to be combined with a data link layer solution. The latter can be classified as a device-oriented physical layer solution.

Figure 1c shows the comparison in terms of energy saving percentage when the ONU architectures option 1 and option 3 in [16] are considered. Option 1 is the traditional ONU receiver architecture with a continuous mode clock and data recovery (CM-CDR) circuit. When the ONU is sleeping, the whole receiver and the SERDES are turned off, while only part of the back-end electronic circuit after the SERDES is kept on. Option 3 features a modified architecture where everything, including the CM-CDR, is kept "on" even during sleep time. The SERDES is turned off, while only part of the back-end electronic circuit is kept on. Option 3 can be considered similar to dozing but without data reception. For both options, $P_{a} \approx 3.85 \mathrm{~W}$. For option $1, P_{s}^{\text {Option1 }} \approx 750 \mathrm{~mW}$ 
and $T_{\text {option } 1}^{\text {Op }} \approx 5.125 \mathrm{~ms}$. For option $3, P_{S}^{\text {Option3 }} \approx 1.28 \mathrm{~W}$ and $T_{\text {oh }}^{\text {Option } 3} \approx 125 \mu \mathrm{s}$. Option 1 has a low $P_{s} / P_{a}$ ratio because many ONU components are turned off when in sleep mode, while it presents high overhead time when waking up from sleeping because the receiver is off. Option 3, conversely, has a higher power ratio than option 1 but less overhead time because clock synchronization is maintained. The analysis considers energy saving performance when the number of ONUs is either 16 or 32 . Figure $1 \mathrm{c}$ shows that option 3 ONU significantly outperforms option 1 ONU when the TDM cycle $T_{c}$ is less than $16 \mathrm{~ms}$ (i.e., $T_{s l}<1 \mathrm{~ms}$ ) when 16 ONUs are present and $T_{s l}<500 \mu$ s when 32 ONUs are present. More significantly, the result also shows that option $1 \mathrm{ONU}$ fails to save energy if the overhead time exceeds the lenght of the TDM cycle. This is because the overhead time exceeds the length of the TDM cycle, which prevents the ONU from switching into sleep mode. Figure 1c also shows that if the sleeping time is large (i.e., the TDM slot size increases as well as the TDM cycle) option 1 saves more energy than option 3 because the detrimental effect of the higher overhead becomes negligible and option 1 consumes less power than option 3. This also shows that data link layer solutions achieve high energy savings when traffic is low (i.e., when the ONU can sleep longer) while they are ineffective when traffic is high because ONUs are forced to sleep less. In the latter case physical layer solutions have higher energy saving potentials.

Figure $2 \mathrm{a}$ shows that the utilization of sleep mode in $1 \mathrm{~Gb} / \mathrm{s}$ TDM EPON with 16 ONUs and with packet size uniformly distributed between 46 and 1500 bytes increases the average end-to-end delay of the downstream traffic. However, results show that the delay is limited to $1 \mathrm{~ms}$ up to very high loads. This value is also the expected waiting time for an upstream frame in a PON with $2 \mathrm{~ms}$ cycle time.

Finally, the feasibility of TDM PON with sleep mode has been experimentally assessed. Figure $2 b$ show the experimental results as also reported in [17]. In the experiment, $O N U^{1}$ implements sleep mode while $O N U$ Uelse emulates the other 15 ONUs. The start of the sleep period is implicit, and it begins with the end of the allocated upstream slot. The end of the sleep period is specified to leave sufficient lead time to account for the synchronization overhead. Figure $2 b$ shows the proper protocol working and that $O N U^{1}$ successfully enters and exits the power save states without either losing traffic or interfering with the upstream transmission from other ONUs.

\section{Conclusion}

Optical access networks are the network segment currently experiencing the highest energy consumption per bit among optical networks. Thus, methods to reduce energy consumption must be promptly provided. In particular, the customer premises (i.e., the ONUs) are the PON segment where savings can be more dramatic. This is mainly due to the high number of deployed ONUs and their current low utilization (i.e., they are often idle).

Several solutions to reduce energy consumption in ONUs have been proposed by many researchers so far. From the quantitative evaluation conducted in this article for TDM PONs with sleep mode, ONUs' energy consumption can be reduced by switching them to low power mode when idle. However, huge savings can only be achieved if (i.e., the how) ONUs are capable of quickly regaining synchronization upon wake up, and the power consumed while sleeping is much less than when the ONU is on. Moreover, data link layer solutions alone (e.g., sleep mode) are effective when network utilization is low, but when network utilization increases, physical layer support (e.g., quick resynchronization) is necessary.

If solutions and ONU architectures with such characteristics are developed, huge energy savings and limited delay increase will be achieved. However, even if energy saving issues in current EPON, 10G-EPON, GPON, and XG-PON are solved, the standardization of next-generation PON (e.g., NG-PON2) will bring more capacity to the end users at the expense of higher power consumption, and thus, new challenges on providing energy efficiency in the optical access will emerge.

\section{Acknowledgment}

The authors would like to thank Andrew Quecan for his help in the patent search.

\section{References}

[1] C. Lange et al., "Energy Consumption of Telecommunication Networks and Related Improvement Options," IEEE J. Sel. Topics in Quantum Electronics, vol. 17, no. 2, Mar.-Apr. 2011.

[2] R. Tucker, "Green Optical Communications - Part II: Energy Limitations in Networks," IEEE J. Sel. Topics in Quantum Electronics, vol. 17, no. 2, Mar.-Apr. 2011

[3] "GPON Power Conservation," ITU-T G-Series Recs. - Supplement 45 (G.sup45), 2009.

[4] E. Trojer and P. Eriksson, "Power saving modes for GPON and VDSL," Proc. 13th Euro. Conf. Net. \& Optical Commun., June 30-July 3, 2008, Krems, Austria.

[5] "Code of Conduct on Energy Consumption of Broadband Equipment," Version 4 ed., European Commission Directorate General Joint Research Center (JRC) Institute for Energy, Renewable Energy Unit, Feb. 102011

[6] K. Christensen et al., "IEEE 802.3az: the Road to Energy Efficient Ethernet," IEEE Commun. Mag., vol. 48, no. 11, Nov. 2010.

[7] S. Nedevschi et al., "Reducing Network Energy Consumption via Sleeping and Rate-Adaptation," Proc. 5th USENIX Symp. Networked Systems Design and Implementation (NSDI), 2008.

[8] J. D. Vereen et al., "Optical Network Terminal with Low-Power Sleep Logic That Substantially Extends the Life of the Battery After the AC Main Power Supply Has Been Lost," Tellabs Petaluma, Inc., Tech. Rep. US007287175B2, Oct. 2007

[9] She-Hwa Yen et al., "Photonic Components for Future Fiber Access Networks," IEEE JSA', vol. 28, no. 6, Aug. 2010.

[10] L. Valcarenghi et al., "Impact of Modulation Formats on ONU Energy Saving," 2010 36th European Conf. and Exhibition on Optical Commun. (ECOC), Sept. 2010.

[11] W. Freude et al.," "Optically Powered Fiber Networks," Optics Express, vol. 16, no. 26, Aug. 2008.

[12] J. Mandin and L. Khermosh, "Power Saving in IEEE 802-Style Networks," US Patent Application, May 13 2010, US201000118753A1.

[13] J. Zhang and N. Ansari, "Toward Energy-Efficient 1G-EPON and 10G-EPON with Sleep-Aware MAC Control and Scheduling," IEEE Commun. Mag., vol. 49, no. 2, Feb. 2011

[14] B. Skubic and D. Hood, "Evaluation of ONU Power Saving Modes for Gigabit-Capable Passive Optical Networks," IEEE Network, vol. 25, no. 2, Mar.-Apr. 2011.

[15] R. Kubo et al. "Study and Demonstration of Sleep and Adaptive Link Rate Control Mechanisms for Energy Efficient 10G-EPON," IEEE/OSA J. Optical Commun. and Networking, vol. 2, no. 9, Sept. 2010, pp. 716-29.

[16] S.-W. Wong et al., "Sleep Mode for Energy Saving PONs: Advantages and Drawbacks," IEEE GLOBECOM Wksps. 2009, Nov. 30-Dec. 4, 2009, Honolulu, Hawaii.

[17] S.-W. Wong et al., "Demonstration of Energy Conserving TDM-PON with Sleep Mode ONU Using Fast Clock Recovery Circuit," IEEE/OSA Optical Fiber Commun. Conf. (OFC) 2010, Mar. 21-25, 2010, San Diego, CA.

\section{Biographies}

LUCA VALCARENGHI [M'00] (luca.valcarenghi@sssup.it) holds a Laurea degree in electronics engineering (1997) from the Politecnico di Torino, Italy, an M.S. in electrical engineering (1999), and a Ph.D. in electrical engineeringtelecommunications (2001) from the University of Texas at Dallas (UTD). Since September 2002 he has been an assistant professor at the Scuola Superiore Sant'Anna, Pisa, Italy. He received a Fulbright Reaserch Scholar Fellowship in 2009 during which he visited the Photonics and Networking Research Laboratory (PNRL) of Stanford University. He has published more than 100 papers in international journals and conference proceedings, and actively participated in the TPC of several IEEE conferences. He has been 
TPC co-chair of the Optical Network and System Symposium at IEEE ICC 2011. His main research interests are optical networks design, analysis, and optimization and energy efficiency in communications networks.

DIVANILSON R. CAMPELO (dcampelo@cin.ufpe.br) is an assistant professor at Centro de Informática ( $\mathrm{Cln}$ ), Federal University of Pernambuco (UFPE), Recife, Brazil. He received his electrical Engineering degree from UFPE in 1998, and Master's and Ph.D. degrees in electrical engineering from the University of Campinas (UNICAMP) in 2001 and 2006, respectively. Before joining CIn/UFPE, he was an assistant professor in the Electrica Engineering Department of the University of Brasilia (UnB). He was also a visiting assistant professor in the Electrical Engineering Department of Stan ford University from September 2008 to December 2009. His research interests are in optical communications, computer networking, telecommunication networks and computer systems.

SHING-WA WONG received the B.S. degree from the Electrical Engineering Department, University of California Los Angeles (UCLA), Los Angeles and a Ph.D. degree in electrical engineering from Stanford University, Stanford, CA. He has also held R\&D positions at AT\&T labs, Qualcomm, and Ericsson. His research interests include broadband access network and optical wireless integration. Since 2011 he is with Ericsson Inc.

SHE-HWA YEN'S bio was not available at the time this issue went to press.

SHINJ YAMASHITA [M'01, SM'11] (shyama@jp.fujitsu.com) received B.S and M.S. degrees in electrical engineering from the University of Tokyo Japan, in 1996 and 1998, respectively. In 1998, he joined Fujitsu Laboratories Ltd. and has been engaged in research and development of optical modules, ICs, MEMS devices, and energy-efficient networks. He is currently interested in research on software defined networks. From 2008 to 2009 he worked as a visiting scholar in the Department of Electrical Engineering at Stanford University. He is a member of the IEEE Communications Society and Photonics Society, and the Institute of Electronics, Information and Communication Engineering (IEICE) of Japan.

DUNG PHAM VAN (d.phamvan@sssup.it) received his B.S. degree in information technology from Hong Duc University, Thanh Hoa, Vietnam, in 2003, and his M.S. degree in information communication technology from GITS, Waseda University, Tokyo, Japan, in 2009. He worked at Hong Duc University as a lecturer from 2004 to 2010. Since 2010, he is pursuing his
Ph.D. in innovative technologies at Scuola Superiore Sant ${ }^{\prime}$ Anna, Pisa, Italy. His current research interests include passive optical networks, energy efficiency in access networks, network management, and traffic analysis.

PIER GIORGIO RAPONI (raponi@sssup.it), received his Laurea degree (B.Eng.) and Laurea Specialistica degree (M.S.), both in telecommunications engineering, from the University of Pisa in 2004 and 2009, respectively. He was an undergraduate student at Scuola Superiore Sant'Anna in Pisa from 2001 to 2008 where he received his Diploma degree in 2004 with honors. He is currently a Ph.D. student at Scuola Superiore Sant'Anna, at the Institute of Technologies for Communication, Information and Perception ( $\mathrm{TeClP}$ ). His research interests include optical interconnection networks for high-performance computing and data centers, energy efficiency in switching architectures, scheduling algorithms, and design and analysis of packet-switched optical networks and nodes.

PIERO CASTOLDI [M'93, SM'12] (castoldi@sssup.it) has been an associate professor in telecommunications and responsible for the networks and services area at Scuola Superiore Sant'Anna since 2001. He holds a Master's degree in electrical engineering from the University of Bologna, Italy, and a Ph.D. degree in information technology from the University of Parma, Italy. He has also been a visiting post-doc researcher at the Department of Electrical Engineering at Princeton University. His scientific activity has covered the areas of digital transmission, telecommunication networks performance, and, more recently, provisioning, protection, and switching techniques for nextgeneration optical networks. He is an author of more than 200 technical papers published in international conferences and journals and of a book on CDMA. He is also a co-inventor of 12 patents.

LEONID G. KAZOVSKY [F] (I.kazovsky@stanford.edu) joined Stanford University in 1990. He founded the Photonics and Networking Research Laboratory (PNRL) at Stanford and has led PNRL since then. The PNRL team includes some 15 researchers working on green optical/wireless access and in-building networks. Prior to joining Stanford, he was with Bellcore doing research on coherent, WDM, and high-speed optical fiber communication systems (later, Bellcore changed its name to Telcordia and was acquired by Ericsson). His research on coherent optical systems is widely considered to be the fundamental foundation of all modern coherent systems. He authored or co-authored three books, three book chapters, 55 invited journal papers and invited conference talks, some 200 journal technical papers, and some 300 conference papers. His latest book is Broadband Optical Access Networks (Wiley, 2011). He is a Fellow of OSA. 